\title{
Pensamento Computacional no Ensino Superior: Relato de uma oficina com professores da Universidade do Vale do Rio dos Sinos
}

\author{
Adriana Justin Cerveira Kampff, Tiago Ricciardi Correa Lopes, Isa Mara da Rosa \\ Alves, Vinicius Costa de Souza, Fernando Pinho Marson, Sandro José Rigo \\ Núcleo de Inovação em Práticas Educacionais \\ Universidade do Vale do Rio dos Sinos (UNISINOS) \\ São Leopoldo - RS - Brasil \\ ackampff@unisinos.br, tricciardi@unisinos.br, ialves@unisinos.br, \\ viniciuscs@unisinos.br, fmarson@unisinos.br, rigo@unisinos.br
}

\begin{abstract}
The article reports a workshop of performing experience on Computational Thinking aimed at higher education teachers. The workshop aimed to sensitize teachers about the application possibilities of this thinking model in educational activities in different areas of knowledge. First, the concept of Computational Thinking is presented as defined by Wing (2006 and 2008). Then the text describes the workshop objectives, the topics and the practical activity performed with the participants, based on a visual framework of organizing procedures for troubleshooting created especially for the occasion of the course. The results of the workshop observed based on a survey of the participants are presented in the last section.
\end{abstract}

Resumo. $O$ artigo relata a realização de oficina sobre Pensamento Computacional destinada a professores do ensino superior. A oficina teve como principal objetivo sensibilizá-los sobre as possibilidades de aplicação deste modelo de pensamento em atividades educacionais de diferentes áreas do conhecimento. $O$ conceito de Pensamento Computacional é apresentado segundo a definição de Wing (2006 e 2008). Em seguida, são descritos os objetivos da oficina, os tópicos abordados e a atividade prática realizada com os participantes, baseada em um framework visual de organização de procedimentos para a solução de problemas, criado especialmente para a ocasião do curso. Os resultados da oficina, observados com base em uma pesquisa com os participantes, são apresentados na última seção.

\section{Introdução}

Na última década, os avanços da web, a emergência dos dispositivos móveis, o crescimento da indústria dos games e o ressurgimento da cultura faça-você-mesmo (doit-yourself), dentre outros fenômenos contemporâneos, evidenciam as carências de conhecimento sobre os modos de funcionamento e mesmo sobre os impactos dos meios computacionais na sociedade e na cultura [Horizon Report, 2016]. Neste contexto, segundo Berrocos et al. (2015), cresce o interesse de governos e instituições públicas e privadas em introduzir e fomentar a aprendizagem de temas relacionados ao ensino de 
programação em todos os níveis escolares. Associado a este movimento em prol da aprendizagem de linguagens de programação propriamente ditas, observa-se também o esforço protagonizado por pesquisadores, educadores e organizações de diversos tipos para fomentar um conjunto de competências, habilidades e disposições relacionadas ao universo da informática, as quais dão forma ao conceito de Pensamento Computacional [Blikstein, 2013].

Para autores como Wing (2006 e 2008), Pensamento Computacional é uma competência analítica fundamental que todas as pessoas, e não somente profissionais de computação, podem usar para resolver problemas em distintos contextos, para projetar sistemas e mesmo para entender o comportamento humano.

Neste contexto, o presente artigo apresenta os resultados alcançados em uma experiência de oficina sobre Pensamento Computacional destinada a professores do ensino superior, realizada durante a Semana Pedagógica da Universidade do Vale do Rio dos Sinos (UNISINOS). O principal objetivo da oficina foi sensibilizar os professores sobre as possibilidades de aplicação deste modelo de pensamento em atividades educacionais relacionadas a variados contextos de aprendizagem. Um dos interesses que motivaram a realização desta oficina foi despertar o interesse e promover o envolvimento de professores de áreas de conhecimento não relacionadas a cursos de Ciências da Computação. Como contribuição adicional, foi desenvolvido um framework visual para facilitar aos participantes a experimentação do processo.

O texto apresenta-se dividido em três seções. Na primeira delas, é descrito o contexto acadêmico e sociocultural em que o conceito de Pensamento Computacional toma forma. Já a segunda seção é destinada à narração do processo que conduziu à realização da oficina de Pensamento Computacional para os professores da UNISINOS. $\mathrm{Na}$ terceira parte, é descrita a atividade prática realizada durante a oficina, através da qual buscou-se aprofundar a compreensão do conceito de Pensamento Computacional, aplicando-se procedimentos orientados à resolução de variados tipos de problemas. Nesta mesma seção é apresentado o "Canvas Algorítmico", um framework visual desenvolvido especialmente para a oficina e que tem como função organizar o processo de criação de procedimentos orientados à resolução de problemas em distintos contextos. Finalmente, na seção de considerações finais, são encaminhadas algumas sugestões de ajuste a partir da experiência relatada.

\section{Pensamento Computacional}

$\mathrm{Na}$ abertura de um artigo inaugural sobre o tema, Jannette Wing (2006), uma pioneira nos estudos sobre Pensamento Computacional, chamava a atenção sobre a importância da aprendizagem de conceitos computacionais. Destacava que não se tratava somente de uma competência cognitiva útil para a escrita de programas informáticos, mas de repertório de conhecimentos favoráveis à resolução de diferentes tipos de problemas, ao estabelecimento de formas de comunicação social e à gestão de múltiplos aspectos da vida cotidiana.

Seguindo por um caminho de argumentação semelhante, para Berrocoso et al. (2015) os processos cognitivos empregados em atividades de programação informática são capazes de fomentar a construção de conhecimentos mediante a apropriação, isto é, mediante resultados que são formas de materializar processos cognitivos e expressivos 
V Congresso Brasileiro de Informática na Educação (CBIE 2016)

Anais dos Workshops do V Congresso Brasileiro de Informática na Educação (CBIE 2016)

dos sujeitos: programas, jogos, produtos hipermídia, robôs e outros objetos computacionais são formas de materialização do pensamento e, portanto, são capazes de simultaneamente modificá-lo e enriquecê-lo.

Perspectivas como estas apresentadas por Berrocoso et al. (2015) e Wing (2006) sustentam a emergência de um tipo específico de abordagem analítica relacionado aos estudos de computação que vem se popularizando através do conceito de Pensamento Computacional. Para Wing (2006) Pensamento Computacional é um termo que designa um conjunto de processos de resolução de diferentes tipos de problemas que incluem características tais como sistematização e análise de dados e criação de soluções que utilizam uma série de passos ordenados (algoritmos).

Segundo Berrocoso et al. (2015), o conceito de Pensamento Computacional diz respeito a uma competência complexa relacionada a um modelo de desenvolvimento de ideias que se aplicam em múltiplos aspectos da vida cotidiana e que possuem como principal característica relacionarem-se simultaneamente com o pensamento abstratomatemático e com o pensamento pragmático-engenheiro. Trata-se, ainda, de uma competência voltada para a resolução de problemas de forma inteligente e imaginativa (qualidades humanas as quais os computadores não detêm), que todo cidadão deveria conhecer para melhor se desenvolver na sociedade digital.

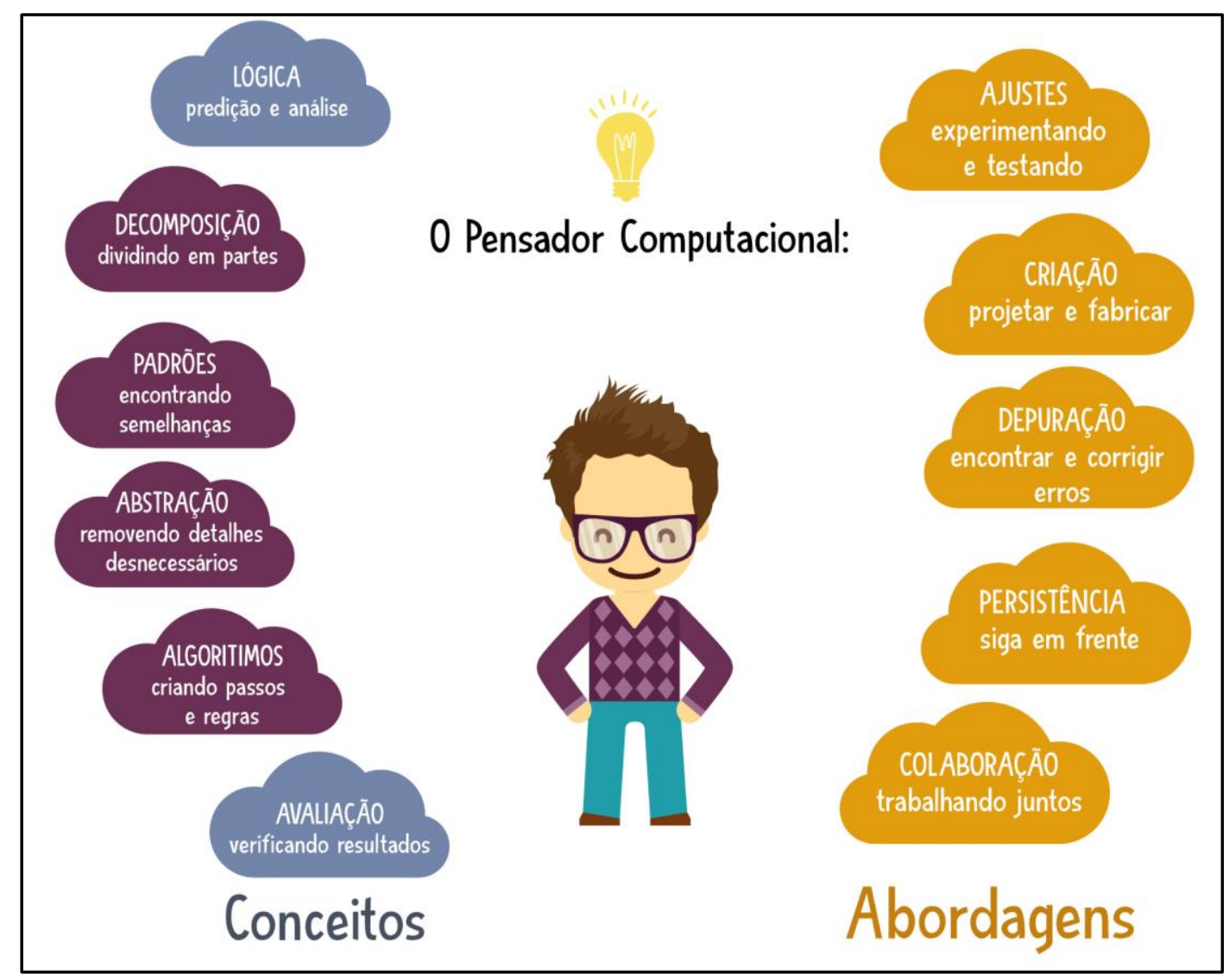

Figura 1. O Pensador Computacional (adaptado de Barefoot Computing, 2016) 
O Pensamento Computacional não diz respeito à atividade de programação de computadores propriamente dita, mas sim à capacidade de pensar abstratamente em diversos níveis, independente dos dispositivos. Pode-se desenvolver o Pensamento Computacional sem computadores - com lápis e papel, por exemplo - ainda que, evidentemente, os computadores auxiliem na resolução de problemas os quais, sem eles, a trajetória até as soluções seria muito mais difícil e, em vários casos, humanamente impossível de se alcançar.

O Pensamento Computacional permite a solução de problemas, decompondo-os em elementos e encontrando algoritmos que os resolvam. Implica em um variado conjunto de procedimentos, dentre os quais destacam-se: decomposição, reconhecimento de padrões, abstração e desenho algorítmico (Figura 1).

Os procedimentos são descritos na sequência:

- Decomposição: A decomposição é um processo de simplificação de um problema complexo através de sua quebra em subproblemas menores.

- Reconhecimento de padrões: o processo de reconhecimento de padrões ocorre na medida em que se consegue reconhecer e agrupar em categorias múltiplas variáveis e dimensões de um determinado problema.

- Abstração: a abstração é a ação de ignorar informações desnecessárias de modo a destacar somente os elementos importantes para conduzir à solução do problema.

- Desenho algorítmico: o desenho algorítmico é a etapa de escrita de um processo que conduza à solução do problema da forma eficiente e utilizandose o menor número de passos possíveis.

Tomando como base os debates em torno do conceito de Pensamento Computacional e as características que lhe conferem propriedades de processo analítico para resolução de problemas em diferentes áreas, um grupo de professores vinculados à gestão dos cursos de graduação da UNISINOS e ao Núcleo de Inovação em Práticas Educacionais (NIPE) propuseram uma oficina de capacitação para o corpo docente da instituição. O objetivo era iniciar um diálogo sobre a importância de adotar alguns dos pressupostos relacionados ao Pensamento Computacional em atividades de sala de aula.

\section{Oficina de Pensamento Computacional}

A UNISINOS é uma instituição de ensino superior privada localizada na cidade de São Leopoldo, Região Metropolitana de Porto Alegre, no Rio Grande do Sul. No período de abertura de todos os semestres letivos, a Universidade promove um conjunto de atividades de formação e capacitação docentes com temáticas variadas, as quais ocorrem durante o período de uma semana e reúnem professores de diversas áreas de conhecimento. Na edição de agosto de 2016, o Pensamento Computacional foi o conceito escolhido como tema central do evento, servindo de base de reflexão e orientação das atividades propostas.

Nesta seção, portanto, é descrito o processo que conduziu à proposição de uma oficina de Pensamento Computacional ofertada para professores de todos os cursos da instituição, na qual reuniram-se mais de cem participantes para debaterem e praticarem conceitos relacionados ao tema central do evento. 
O primeiro desafio encontrado pela equipe de Formação Docente, que trabalhou em parceria com o NIPE, foi encontrar uma forma de projetar uma atividade de Pensamento Computacional que conseguisse engajar professores de áreas de conhecimento não relacionadas à Ciência da Computação. Foram realizadas várias reuniões para debater o planejamento da oficina, bem como as possibilidades de estratégias adotadas para tornar a abordagem sobre o tema ao mesmo tempo acessível e familiar para todo o grupo de professores. Em determinado momento do processo, optou-se por realizar um teste de apresentação dos conteúdos selecionados para serem trabalhados durante a oficina com professores representantes das diferentes áreas de conhecimento, tendo em vista verificar a receptividade às discussões teóricas, exemplos apresentados e atividades propostas. Este encontro foi fundamental para que algumas decisões fossem tomadas, descritas na sequência.

Em primeiro lugar, foi proposto que, embora adotando-se a mesma estrutura de tópicos trabalhados, a oficina deveria apresentar versões diferentes, orientadas para distintos perfis de professores, os quais foram agrupados segundo suas áreas de atuação. Baseando-se nos dados obtidos a partir das inscrições realizadas no evento e tomandose como base a divisão institucional adotada pela UNISINOS ${ }^{1}$, decidiu-se por organizar as turmas de professores em quatro grupos: Grupo 1: composto por professores da Escola Politécnica juntamente àqueles que atuam na Escola de Gestão e Negócios; Grupo 2: professores da Escola da Indústria Criativa; Grupo 3: Escolas de Humanidades e Direito; Grupo 4: Escola de Saúde.

Em segundo lugar, e como decorrência da decisão anterior, foi acordado entre os integrantes da equipe organizadora que os exemplos ilustrativos apresentados durante a oficina deveriam ser selecionados de modo a tentar aproximá-los o máximo possível dos cotidianos acadêmico e profissional correspondentes a cada uma das áreas de conhecimento representadas pelos grupos heterogêneos de participantes.

Por fim, o grupo de professores responsáveis pelo planejamento da oficina definiu que o planejamento deveria contemplar três etapas: a) debate sobre a presença ubíqua da computação em nossa sociedade a partir de exemplos selecionados por áreas de conhecimento; b) apresentação do conceito de Pensamento Computacional; c) realização de uma atividade prática com os participantes da oficina. Sobre esta última etapa, um ponto de consenso foi que a atividade deveria aprofundar alguns dos aspectos conceituais relacionados ao Pensamento Computacional por meio de um exercício que pudesse ser realizado em pequenos grupos e sem o uso de computadores. A partir destes pressupostos, foi desenvolvido um mapa visual de organização de procedimentos para a solução de problemas, o Canvas Algorítmico, que será descrito na próxima seção.

\subsection{Canvas Algorítmico}

Tendo em vista a realização de uma atividade prática com os participantes da oficina, que pudesse ser realizada em grupos e sem o uso de computadores, foi desenvolvido um modelo canvas baseado em um conjunto de procedimentos para resolução de problemas, tomando-se como referência alguns dos pressupostos do Pensamento Computacional (Figura 2).

\footnotetext{
${ }^{1}$ Humanidades, Saúde, Indústria Criativa, Gestão e Negócios, Direito, Politécnica.
} 
V Congresso Brasileiro de Informática na Educação (CBIE 2016)

Anais dos Workshops do V Congresso Brasileiro de Informática na Educação (CBIE 2016)

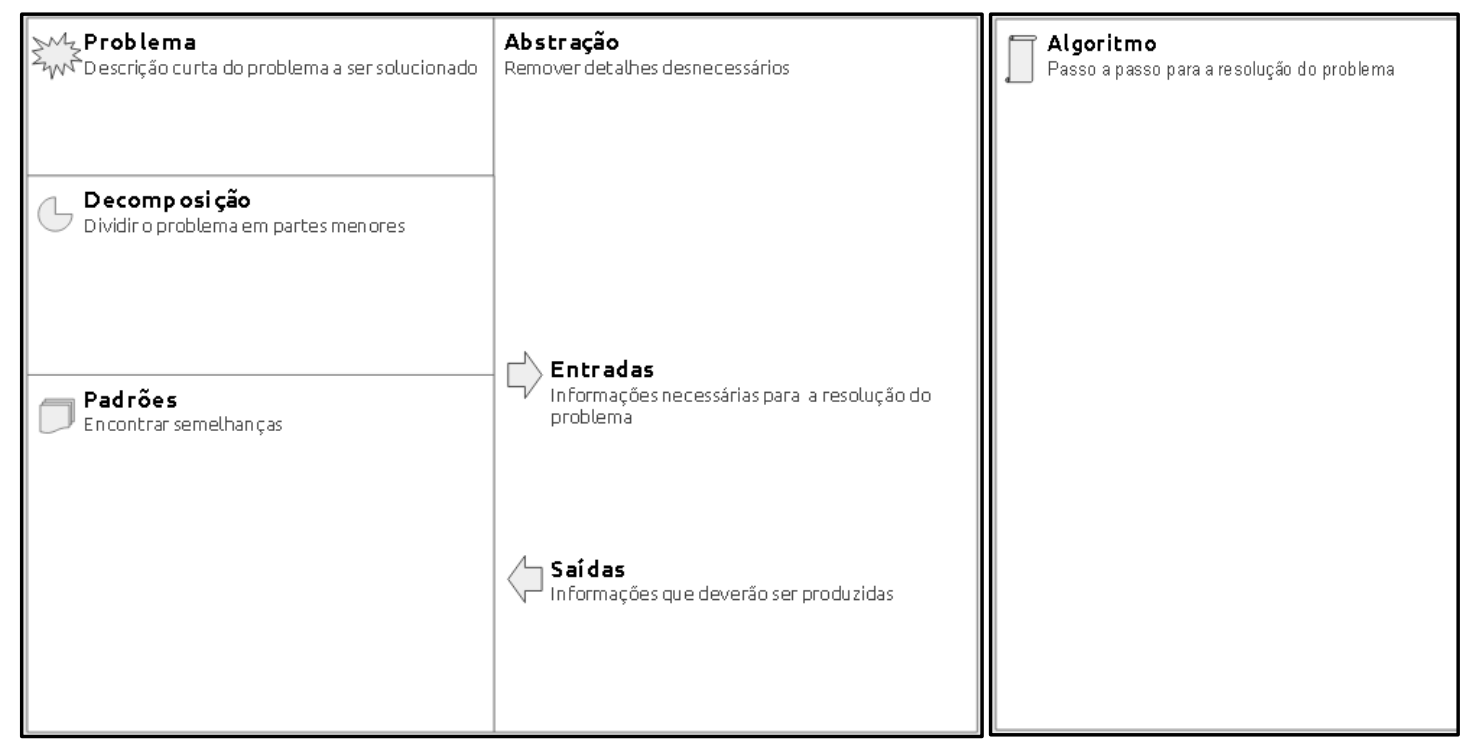

Figura 2. Canvas Algorítmico (fonte: autores)

Partindo-se de um dado problema, o canvas tem como propósito principal orientar aqueles que o utilizam através de uma série de passos que conduzam até sua resolução. Os passos retomam os principais procedimentos relacionados ao Pensamento Computacional, são eles: decomposição, padrões, abstração e desenho algorítmico. Assim, após serem discutidos exemplos de recursos computacionais utilizados no meio acadêmico e profissional, seguido da discussão sobre os processos presentes no Pensamento Computacional, apresentou-se o Canvas Algorítmico e exemplos em diversas áreas do conhecimento. A título de exemplo, apresentamos, na Figura 3, uma forma de como o canvas poderia ser preenchido a partir do problema "Como melhorar a coleta de lixo de uma cidade"?

Após a discussão dos exemplos, em grupos de 4 a 5 pessoas, os docentes pensaram em problemas complexos de suas áreas de atuação e utilizaram o Canvas Algorítmico para propor soluções. O material gerado foi partilhado com os demais grupos presentes em cada uma das oficinas. Como as quatro oficinas ocorreram simultaneamente, no primeiro dia da Semana Pedagógica da UNISINOS, os professores seguiram discutindo suas possibilidades para uma nova cultura universitária, ratificando a importância de oportunizar formação aos estudantes universitários sobre Pensamento Computacional, o que já ocorrerá neste semestre por meio da oferta de cursos de extensão.

Ao final da Semana Pedagógica, os professores participantes foram demandados a responder uma pesquisa sobre as atividades realizadas. Foram questionados aspectos referentes à dinâmica do encontro, à aplicabilidade e uma avaliação geral, em que cada questão tinha como possibilidade de resposta os conceitos ótimo, bom, regular ou insuficiente. A pesquisa de avaliação foi respondida por 53 professores. Sobre a dinâmica das oficinas, $68 \%$ dos respondentes atribuiu conceito ótimo e 32\% indicou como bom. Em relação à aplicabilidade, 66\% considerou ótimo, 30\% classificaram como bom e apenas $4 \%$ (dois respondentes da área da saúde) indicaram como regular. Quanto à avaliação geral, $60 \%$ dos respondentes classificaram a oficina como ótima e $40 \%$ deles como boa. 
V Congresso Brasileiro de Informática na Educação (CBIE 2016)

Anais dos Workshops do V Congresso Brasileiro de Informática na Educação (CBIE 2016)

\begin{tabular}{|c|c|}
\hline $\begin{array}{l}\text { Problema } \\
\text { Melhosar a coleta de lixo de uma cidade } \\
\text { (coletar mais lixo em menos tempo, percorrendo a } \\
\text { menor distância) }\end{array}$ & \multirow{3}{*}{$\begin{array}{l}\text { Abstração } \\
\text { Remover detalhes desnecessários } \\
\text { Lixeiras: } \\
\text { - possuem diferentes capacidades } \\
\text { Rotas: } \\
\text { - possuem distância } \\
\text { - duram determinado tempo } \\
\text { - possuem diferentes condiçōes de trânsito } \\
\text { Entradas } \\
\text { Informaçôes necessárias para a resolução do } \\
\text { problema } \\
\text { Localização das lixeiras } \\
\text { Mapa da cidade } \\
\text { Quantidade de lixo dentro das lixeiras } \\
\text { Quantidade de coletores de lixo } \\
\text { Capacidade de cada coletor de lixo } \\
\text { Condiçós de trânsito em cada horário } \\
\text { Horários de início da coleta } \\
\\
\text { Saídas } \\
\text { Informaçōes que deverão ser produzidas } \\
\text { Rota otimizada para cada um dos coletores disponiveis } \\
\text { comm o tempo previsto e a quantidade de lixo a ser } \\
\text { coletada. }\end{array}$} \\
\hline $\begin{array}{l}\text { Decomposição } \\
\text { Quebrar o problema em partes } \\
\text { 1. Como saber quais lixeiras precisam de coleta? } \\
\text { 2. Como definir a melhor rota para coletar o lixo } \\
\text { necessário? }\end{array}$ & \\
\hline $\begin{array}{l}\text { Padrōes } \\
\text { Encontrar semelhanças genéricas } \\
\text { Lixeiras: } \\
\text { - são de plástico, alumínio, lata, ferro, etc } \\
\text { - possuem differentes cores } \\
\text { - possuem formato coniesco, retangular, quadrado } \\
\text { - possuem capacidade de } 50 \text { a } 5000 \text { litros } \\
\text { - exclusivas para vidros, latas, orgânicos, etc } \\
\text { Rotas: } \\
\text { - as rotas possuem distância entre } 50 \text { e } 500 \mathrm{~km} \\
\text { - otempo de cada rota varia entre } 1 \text { e } 8 \text { horas } \\
\text { - cada a rota atende um conjunto de bairros } \\
\text { - cada rota possui diferentes condiçôes de } \\
\text { trânsito }\end{array}$ & \\
\hline \multicolumn{2}{|c|}{$\begin{array}{l}\text { Algoritmo } \\
\text { Passo-a-passo para a resolução do problema }\end{array}$} \\
\hline \multicolumn{2}{|c|}{ 1. Identificar quais lixeiras possuem lixo. } \\
\hline \multicolumn{2}{|c|}{ 2. Obter a localização dessas lixeiras. } \\
\hline \multicolumn{2}{|c|}{ 3. Calcular todas as rotas possíveis que passem por essas lixeiras } \\
\hline \multicolumn{2}{|c|}{ 4. Calcular a quantidade necessária de coletores. } \\
\hline \multicolumn{2}{|c|}{ 5. Selecionar a melhor rota para cada coletor. } \\
\hline 6. Calcular estimativa de temp & o para a rota \\
\hline 7. Calcular a quantidade de lix & o a ser coletada em cada rota. \\
\hline
\end{tabular}

Figura 3. Exemplo Preenchido do Canvas Algorítmico (fonte: autores)

Ao avaliarmos os dados da pesquisa, verifica-se que os professores foram bastante receptivos à proposta, reconheceram o alto grau de aplicabilidade dos conceitos explorados e demonstraram compreender a importância do desenvolvimento do Pensamento Computacional na Universidade. O engajamento dos docentes durante as oficinas ratificou o compromisso do NIPE em oportunizar novas formações sobre o tema, aprofundando conceitos e aplicações.

\section{Considerações finais}

Assim como nossos antepassados se viram diante do desafio de promover a popularização do domínio da linguagem verbal escrita entre as comunidades de suas 
V Congresso Brasileiro de Informática na Educação (CBIE 2016)

Anais dos Workshops do V Congresso Brasileiro de Informática na Educação (CBIE 2016)

épocas e mais recentemente passamos a nos tornar mais familiarizados com os processos produtivos e os códigos de linguagem das mídias audiovisuais, atualmente nossa civilização tem diante de si o desafio de também tornar-se alfabetizada nas linguagens de criação de produtos digitais, tendo em vista que o mundo que nos cerca apresenta-se dominado pelas tecnologias computacionais: desde os recursos mais básicos necessários para a sobrevivência até aqueles voltados para o trabalho, para a comunicação interpessoal, para o desenvolvimento intelectual ou simplesmente para o entretenimento, todos, sem exceção, são controlados em diversos níveis por softwares.

Cabe então assumirmos este estado das coisas e, no que tange especificamente às atribuições das instituições educacionais, propor meios de promover o acesso ao conhecimento que emancipe as pessoas da condição de meros usuários das interfaces digitais para auxiliá-las a tornarem-se programadoras dessas interfaces. Aprender a programar para não ser programado é também, e antes de tudo, uma posição ética frente às novas formas de detenção do conhecimento e gestão da distribuição de poderes nas sociedades contemporâneas.

\section{Referências}

Barefoot Project (2014). "Computational Thinking”. Disponível em: http://barefootcas.org.uk/barefoot-primary-computing-

resources/concepts/computational-thinking/. Acesso em 22 de agosto de 2016.

Berrocoso, J., Sánchez, M., Arroyo, M. (2015). "El pensamiento computacional y las nuevas ecologías del aprendizage". RED-Revista de Educación a Distancia, 46(3).

Blikstein, P. (2013). "Digital Fabrication and 'Making' in Education: The Democratization of Invention". In J. Walter-Herrmann \& C. Büching (Eds.), FabLabs: Of Machines, Makers and Inventors. Bielefeld: Transcript Publishers. Disponível em: https://tltl.stanford.edu/sites/default/files/files/documents/ publications/2013.Book-B.Digital.pdf. Acesso em 22 de agosto de 2016.

Horizon Report (2016). NMC Horizon Report: Higher Education Edition. Disponível em: http://www.nmc.org/publication/nmc-horizon-report-2016-higher-educationedition/. Acesso em 22 de agosto de 2016.

Wing, J. M. (2006). “Computational Thinking”. ACM 49 Viewpoint, pp. 33-35.

Wing, J. M. (2008). "Computational thinking and thinking about computing". Philosophical Transactions of The Royal Society. 366, pp. 3717-3725. Disponível em: http://www.cs.cmu.edu/ wing/publications/Wing08a.pdf. Acesso em 22 de agosto de 2016. 